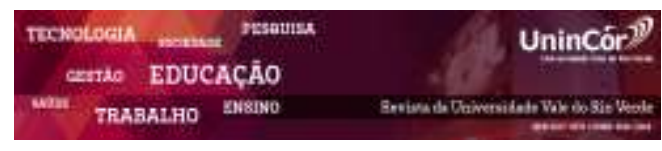

Revista da Universidade Vale do Rio Verde ISSN: 1517-0276 / EISSN: 2236-5362 Vol. 16 | n. 1 | Ano 2018

Letícia Carvalho Massaroli Centro Universitário das Faculdades Associadas de Ensino- UNIFAE leticiamassaroli@gmail.com

Letícia Cristina Santos Centro Universitário das Faculdades Associadas de Ensino- UNIFAE

leticiacristinas@hotmail.com

Giovanna Gomes Carvalho

Centro Universitário das Faculdades Associadas de Ensino- UNIFAE gicrvalho@hotmail.com

Stephanie Avesani João Figueiredo Carneiro

Centro Universitário das Faculdades Associadas de Ensino- UNIFAE stephanieavesani@yahoo.com.br

Laura Ferreira de Rezende Universidade de Campinas e Universidade do Estado de São Paulo laura@fae.br

\section{QUALIDADE DE VIDA E O IMC ALTO COMO FATOR DE RISCO PARA DOENÇAS CARDIOVASCULARES: REVISÃO SISTEMÁTICA}

\section{RESUMO}

Objetivo: As doenças cardiovasculares são as principais causas de morte no Brasil. Têm-se estudado a importância dos fatores de risco para a prevalência da patologia. O objetivo do presente estudo foi revisar artigos para relacionar o IMC alto como fator de risco para doenças cardiovasculares, atentando para a importância da qualidade de vida para prevenção de doenças. Métodos: Foram pesquisados artigos nas bases de dados Lilacs, Scielo e Embase. Foram consideradas as características gerais dos estudos como fatores de risco para doenças cardiovasculares, IMC alto, hipertensão arterial e sedentarismo. Resultados: Dos 77 artigos encontrados, 16 atenderam aos critérios de inclusão. As prevalências das doenças cardiovasculares, como hipertensão arterial, aumentaram com a idade e com a massa corporal. Os resultados apontaram que o sedentarismo também leva a alterações pressóricas. A prevalência entre os sexos foi variável. Conclusão: O IMC alto é um importante fator de risco para doenças cardiovasculares e associado a outros fatores como a idade avançada, tabagismo, hipertensão arterial, níveis elevados de LDL, HDL e triglicerídeos, sedentarismo, etc. aumentam as chances de ocorrências de DCV. A qualidade de vida e os fatores que a influenciam são de suma importância para o controle dos níveis pressóricos. É importante que os programas de saúde atentem para a promoção de saúde e prevenção de DCV.

Palavras chave: IMC. Doença Cardiovascular. Epidemiologia. Qualidade de Vida. Fator de Risco.

\section{QUALITY OF LIFE AND THE HIGH BMI AS RISK FACTOR FOR CARDIOVASCULAR DISEASES: SYSTEMATIC REVIEW}


Conclusion: the IMC is an important risk factor for cardiovascular disease and associated with other factors such as older age, smoking, high blood pressure, high levels of LDL, HDL and triglycerides, sedentary lifestyle, etc. increase the chances of occurrence of CVD. The quality of life and the factors that influence are of paramount importance for the control of blood pressure. It is important that the health programs pay attention to the promotion of health and prevention of CVD.

Keywords: BMI. Cardiovascular Disease. Epidemiology. Quality of life. Risk factor.

Recebido em: 18/11/2017 - Aprovado em: 16/02/2018 - Disponibilizado em: 15/07/2018

\section{INTRODUÇÃO}

As doenças cardiovasculares sãs as principais causas de morte no Brasil. Têm-se estudado, principalmente, a relação entre o estilo de vida e as manifestações cardiovasculares. Segundo a Federação Mundial do Coração (World Heart Federation, 2010) são destacados alguns fatores de risco para doenças cardiovasculares (DCV) como: sobrepeso/obesidade (IMC alto, circunferência abdominal elevada), sedentarismo, estresse, histórico familiar de DCV e fatores genéticos. Além desses fatores, são apontados outros estudos que identificaram diferentes fatores de risco como: hipertensão arterial, tabagismo, idade, diabetes mellitus, níveis alterados de HDL-colesterol (OLIVEIRA et al, 2009)

Dados Epidemiológicos do DataSUS, levantados em 2011, apontaram para um aumento de $196 \%$ em relação ao levantamento de 2001, em que houveram 808 óbitos por obesidade. Segundo o Ministério da Saúde, no Brasil, pelo menos $17 \%$ da população é obesa devido ao sedentarismo e ao alto consumo de produtos industrializados. O índice de massa corporal (IMC) é considerado a técnica mais usada para diagnosticar obesidade por ser simples e ser passível de comparação. Estudos epidemiológicos demonstraram uma clara relação entre IMC elevado e risco de mortalidade cardiovascular (PAOLINO et al, 2010). O objetivo do presente estudo foi revisar artigos para relacionar o IMC alto como fator de risco para doenças cardiovasculares, atentando para a importância da qualidade de vida para prevenção de doenças.

\section{MÉTODOS}

A revisão sistemática foi feita nas bases de dados Lilacs, Scielo e Embase, usando as palavras chaves: IMC e doença cardiovascular, e epidemiologia doenças cardiovasculares. Foram inclusas pesquisas que tratassem sobre IMC elevado, fatores de risco para doenças cardiovasculares, hipertensão arterial sistêmica, sedentarismo e artigos produzidos de 2008 em diante. Artigos de revisão e artigos que abordaram assuntos como doença coronariana crônica, crianças, idosos acamados e com outras doenças além de doenças cardiovasculares, e complicações no pós-operatório foram excluídos da revisão. (Figura 1) 


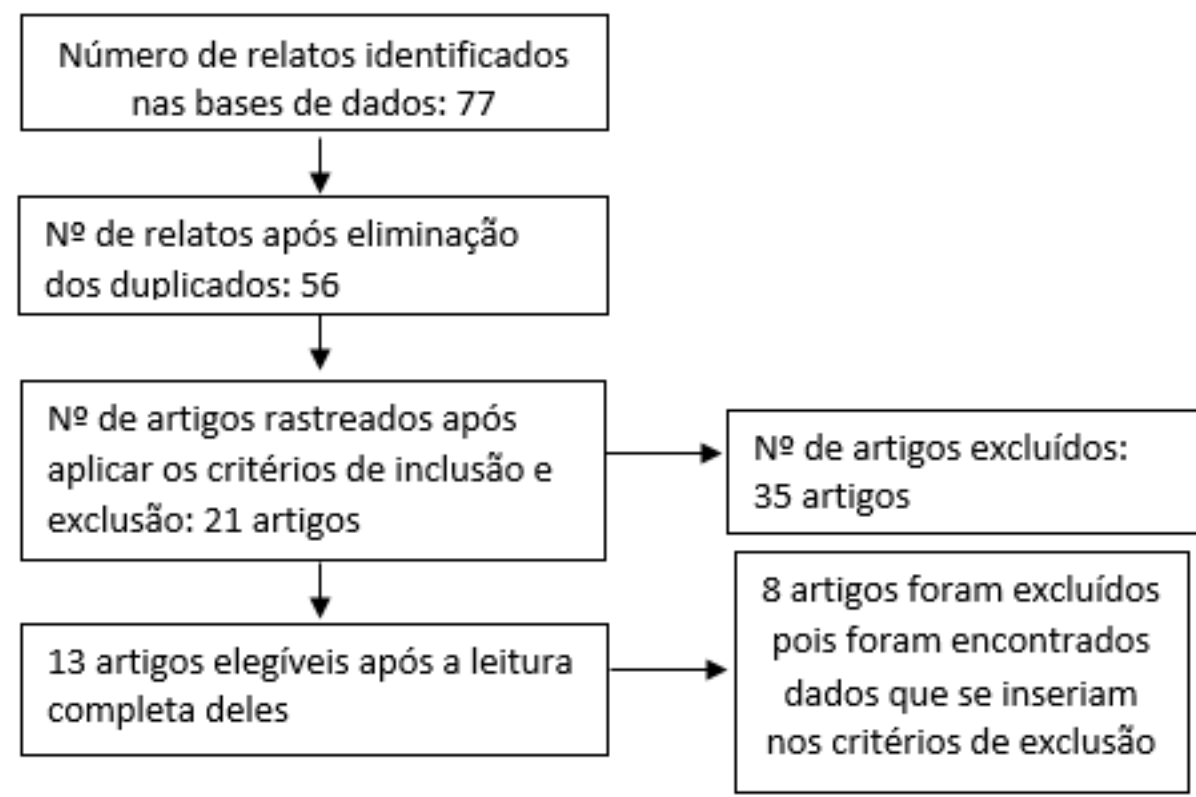

Figura 1 - Fluxograma com embasamento nos dados fornecidos pelos artigos científicos

\section{RESULTADOS E DISCUSSÃO}

Foram selecionados 16 artigos originais

publicados a partir do ano de 2008. (Tabela 1) 


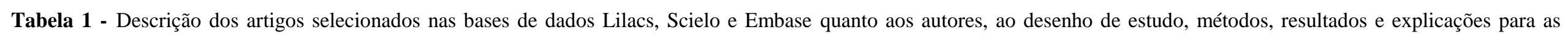
inferências.

\begin{tabular}{|c|c|c|c|c|}
\hline Autor & Método & Avaliação & Resultados & $\begin{array}{c}\text { Explicação possível para os resultados } \\
\text { encontrados }\end{array}$ \\
\hline $\begin{array}{l}\text { Audi et al, } \\
2016\end{array}$ & $\begin{array}{l}\text { Estudo transversal de } \\
127 \text { trabalhadores de } \\
\text { uma penitenciária } \\
\text { feminina do interior de } \\
\text { São Paulo. }\end{array}$ & $\begin{array}{l}\text { Questionários estruturados que } \\
\text { evidenciavam as condições } \\
\text { socioeconômicas, demográficas e } \\
\text { fatores de risco para DCV com } \\
\begin{array}{ll}\text { aferição das } & \text { medidas } \\
\text { antropométricas. } & \text { Exames } \\
\text { bioquímicos e clínicos. }\end{array}\end{array}$ & $\begin{array}{l}\text { Maiores proporções de circunferência da cintura acima dos } \\
\text { valores recomendados }(58,1 \%) \text { e de acúmulo de dois fatores } \\
\text { de risco, no sexo feminino }(33,8 \%) \text {; entre os homens, maior } \\
\text { prevalência de níveis alterados de glicose e de pressão } \\
\text { arterial. }\end{array}$ & $\begin{array}{l}\text { Fatores de risco para DCV encontrados } \\
\text { nos servidores avaliados: circunferência } \\
\text { de cintura e níveis da glicose e pressão } \\
\text { arterial acima dos valores recomendados; } \\
\text { esses fatores podem ser enfrentados com } \\
\text { medidas de promoção e prevenção de } \\
\text { agravos e atenção àqueles que já se } \\
\text { encontram doentes. }\end{array}$ \\
\hline $\begin{array}{l}\text { Carletti et } \\
\text { al, } 2008\end{array}$ & $\begin{array}{l}104 \text { adolescentes, } \\
\text { divididos nos grupos de } \\
\text { sobrepeso/obesos } \\
\text { (GSO) e eutróficos } \\
\text { (GE). }\end{array}$ & $\begin{array}{l}\text { Aferição de variáveis } \\
\text { antropométricas (peso, estatura e } \\
\text { IMC), composição corporal (dobra } \\
\text { cutânea) e variáveis } \\
\text { hemodinâmicas de PAS e PAD e } \\
\text { FC, no repouso e no esforço } \\
\text { máximo do teste cardiopulmonar. }\end{array}$ & $\begin{array}{l}\text { Homens: maiores valores de PAS de repouso para o GSO, } \\
\text { quando comparados com o GE }(113 \pm 13 \text { vs } 106 \pm 8 \\
\text { mmHg), a PAS pré-exercício }(120 \pm 14 \text { vs } 109 \pm 10 \mathrm{mmHg}) \\
\text { e de PAS na carga máxima de trabalho }(156 \pm 20 \text { vs } 146 \pm \\
14 \mathrm{mmHg}) \text {. Mulheres: apenas a PAS pré-exercício foi } \\
\text { superior no grupo de sobrepeso, quando isso foi comparado } \\
\text { com as eutróficas ( } 114 \pm 11 \text { vs } 106 \pm 10 \mathrm{mmHg} \text {.). }\end{array}$ & $\begin{array}{l}\text { Resposta pressórica durante o exercício } \\
\text { foi mais exacerbada em adolescentes } \\
\text { obesos quando comparada com àquela } \\
\text { obtida em eutróficos, indicando maior } \\
\text { reatividade ao estresse físico. }\end{array}$ \\
\hline $\begin{array}{l}\text { Christofaro } \\
\text { et al, } 2011\end{array}$ & $\begin{array}{l}\text { Estudo transversal com } \\
1.021 \text { adolescentes }\end{array}$ & $\begin{array}{l}\text { Questionários que determinem } \\
\text { condição econômica, verificação } \\
\text { das medidas antropométricas e } \\
\text { aferição da PAS. }\end{array}$ & $\begin{array}{l}\text { Menor prevalência de sedentarismo e maiores prevalências } \\
\text { de obesidade abdominal e de sobrepeso entre adolescentes } \\
\text { da classe econômica alta, com maior fator de risco para } \\
\text { DCV. Não houve diferença de prevalência de PAS elevada } \\
\text { entre adolescentes da classe econômica baixa e alta. }\end{array}$ & $\begin{array}{l}\text { Prevalência de fatores de risco para DCV } \\
\text { foi maior na classe econômica alta, exceto } \\
\text { sedentarismo e PAS elevada. }\end{array}$ \\
\hline $\begin{array}{l}\text { Coltro et } \\
\text { al, } 2009\end{array}$ & $\begin{array}{l}\text { Estudo transversal de } \\
428 \text { fichas de } \\
\text { voluntários } \\
\text { participantes de evento } \\
\text { em saúde, }\end{array}$ & $\begin{array}{l}\text { Entrevista feita por estudantes de } \\
\text { medicina com informações sobre } \\
\text { antecedentes pessoais e familiares, } \\
\text { condições de vida e hábitos e para } \\
\text { o conhecimento dos fatores de } \\
\text { risco cardiovascular. Medidos } \\
\text { peso e altura, aferição da PAS e } \\
\text { glicemia. }\end{array}$ & $\begin{array}{l}\text { Idade média de } 57 \pm 14 \text { anos. Principais fatores de risco } \\
\text { cardiovascular: HAS, DM e dislipidemia. Obesos: PAS, } \\
\text { PAD e a glicemia casual maiores que nos não obesos. } \\
\text { Associação entre obesidade e dislipidemia. }\end{array}$ & $\begin{array}{l}\text { Necessidade de medidas de saúde pública } \\
\text { que promovam educação e prevenção } \\
\text { primária dirigida, principalmente, para } \\
\text { indivíduos idosos e com sobrepeso }\end{array}$ \\
\hline
\end{tabular}




\begin{tabular}{|c|c|c|c|c|}
\hline $\begin{array}{l}\text { Espinosa et } \\
\text { al, } 2015\end{array}$ & $\begin{array}{l}\text { Estudo transversal com } \\
\text { dois grupos de idosas. } \\
\text { O primeiro com } 305 \\
\text { idosas e o segundo com } \\
50 \text { idosas praticantes de } \\
\text { exercícios físicos. }\end{array}$ & $\begin{array}{l}\text { Avaliados: } \\
\text { abdominal } \text { IMC, perímetro } \\
\text { (PA), perímetro da } \\
\text { cintura } \quad \text { (PC), } \quad \text { razão } \\
\text { abdômen/quadril (RAQ), razão } \\
\text { cintura/quadril (RCQ), índice de } \\
\text { forma corporal (IFC) e gordura } \\
\text { corporal. }\end{array}$ & $\begin{array}{l}\text { O GEF apresentou valores menores que o EMIPOA para: } \\
\text { MC, IMC, PA, PC, RAQ, RCQ e IFC. Estatura, somatório } \\
\text { de dobras cutâneas e a gordura corporal não diferiram. Para } \\
\text { o estrato } 70-79 \text { anos, apenas foi encontrada diferença para a } \\
\text { estatura (GEF>EMIPOA). }\end{array}$ & $\begin{array}{l}\text { A participação em programa de exercício } \\
\text { físico influenciou os níveis dos } \\
\text { indicadores antropométricos de risco } \\
\text { cardiovascular, e esta influência foi } \\
\text { atenuada nos indivíduos mais velhos. }\end{array}$ \\
\hline $\begin{array}{l}\text { Fonseca et } \\
\text { al, } 2009\end{array}$ & $\begin{array}{l}\text { Estudo transversal com } \\
16 \text { mulheres }\end{array}$ & $\begin{array}{l}\text { Medidas antropométrica, IMC. } \\
\text { Análise de variáveis bioquímicas } \\
\text { plasmáticas como LDL, HDL, } \\
\text { TRIG, colesterol total e glicemia } \\
\text { de jejum. }\end{array}$ & Todas as mulheres apresentaram obesidade abdominal & $\begin{array}{l}\text { Obesidade andrógena pode trazer } \\
\text { alterações no metabolismo de lipídios } \\
\text { revela-se necessário uma intervenção } \\
\text { terapêutica a fim de reduzir estes fatores } \\
\text { de risco. Visa proporcionar uma melhor } \\
\text { qualidade de vida a estes indivíduos, com } \\
\text { programas de atividade física ou } \\
\text { juntamente com o uso de medicamentos. }\end{array}$ \\
\hline $\begin{array}{l}\text { Lavrador et } \\
\text { al, } 2010\end{array}$ & $\begin{array}{l}\text { Estudo transversal com } \\
\text { amostra inicial de } 2.330 \\
\text { adolescentes e amostra } \\
\text { final de } 80 \text { adolescentes } \\
\text { obesos. Divididos em } \\
\text { dois grupos de acordo } \\
\text { com o grau de } \\
\text { obesidade. }\end{array}$ & $\begin{array}{l}\text { IMC, avaliação antromométrica, } \\
\text { aferição da pressão arterial e } \\
\text { avaliação da composição corporal } \\
\text { pelo DXA. }\end{array}$ & $\begin{array}{l}\text { Quanto maior o grau da obesidade, maior o risco de haver } \\
\text { alterações glicídicas, lipídicas e de pressão arterial. }\end{array}$ & $\begin{array}{l}\text { Maior grau de obesidade teve associação } \\
\text { positiva e estatisticamente significante } \\
\text { com a frequência desses fatores de risco: } \\
\text { obesidade, hipertrigliceridemia, baixo } \\
\text { HDL, HAS e algum parâmetro } \\
\text { relacionado ao perfil glicídico, neste } \\
\text { estudo, a glicemia de jejum. }\end{array}$ \\
\hline $\begin{array}{l}\text { Mansur et } \\
\text { al, } 2016\end{array}$ & $\begin{array}{l}\text { Padronização direta da } \\
\text { população de } 2000 . \\
\text { Analisou-se tendências } \\
\text { da mortalidade por } \\
\text { DCV, DIC e DCbV em } \\
\text { mulheres e homens com } \\
\geq 30 \text { anos de idade nas }\end{array}$ & $\begin{array}{l}\text { Os dados sobre a mortalidade } \\
\text { foram obtidos no portal do } \\
\text { Ministério da Saúde. Os dados } \\
\text { populacionais do Instituto } \\
\text { Brasileiro de Geografia e } \\
\text { Estatística (IBGE) foram obtidos } \\
\text { no mesmo portal. }\end{array}$ & $\begin{array}{l}\text { Mortalidade por DCV: redução nas regiões Norte, Centro- } \\
\text { Oeste, Sul e Sudeste; aumento na região Nordeste; DIC: } \\
\text { redução nas regiões Sudeste e Sul; aumento na região } \\
\text { Nordeste; e inalterada nas regiões Norte e Centro-Oeste; } \\
\text { DCbV: redução nas regiões Sul, Sudeste e Centro-Oeste; } \\
\text { aumento na região Nordeste; e inalterada na região Norte. }\end{array}$ & $\begin{array}{l}\text { Permanência da mortalidade nas cinco } \\
\text { regiões do Brasil levará em poucos anos a } \\
\text { inversão do risco de morte entre as } \\
\text { regiões, tornando a região Nordeste e, em } \\
\text { menor grau, as regiões Norte e Centro- } \\
\text { Oeste, as regiões com maiores } \\
\text { coeficientes de mortalidade por DCV. }\end{array}$ \\
\hline
\end{tabular}




\begin{tabular}{|c|c|c|c|c|}
\hline & $\begin{array}{l}\text { cinco regiões do Brasil } \\
\text { no período de 1980- } \\
2012 \text {. }\end{array}$ & & & \\
\hline $\begin{array}{l}\text { Mansur et } \\
\text { al, } 2012\end{array}$ & $\begin{array}{l}\text { Dados populacionais e } \\
\text { de mortalidade foram } \\
\text { obtidos do IBGE e do } \\
\text { Ministério da Saúde. } \\
\text { Risco de morte foi } \\
\text { ajustado pelo método } \\
\text { direto, tendo como } \\
\text { referência a população } \\
\text { mundial de } 2000 \text {. }\end{array}$ & $\begin{array}{l}\text { Regressão linear simples foi usada } \\
\text { para analisar a evolução temporal } \\
\text { da taxa de mortalidade associada à } \\
\text { DIC e à DCbV, e realizou-se a } \\
\text { comparação das inclinações das } \\
\text { linhas de regressão. Software } \\
\text { estatístico utilizado foi o Primer of } \\
\text { Biostatistics, versão 4.02.9. }\end{array}$ & $\begin{array}{l}\text { Redução do risco de morte por DIC e por DCbV no Brasil e } \\
\text { na RMSP. De } 1990 \text { a } 2009 \text {, constatou-se redução da } \\
\text { mortalidade por DIC e DCbV em mulheres e homens no } \\
\text { Brasil e na RMSP. Observou-se maior redução da } \\
\text { mortalidade por DIC nos homens na RMSP do que no } \\
\text { Brasil e nas mulheres na RMSP. Foi registrada maior } \\
\text { redução da mortalidade por DCbV nos homens na RMSP } \\
\text { que no Brasil e igual redução nas mulheres na RMSP e no } \\
\text { Brasil. A redução da mortalidade foi significativa para todas } \\
\text { as faixas etárias analisadas. }\end{array}$ & $\begin{array}{l}\text { Redução na mortalidade por DCV, DIC e } \\
\text { DCbV no Brasil e na RMSP. Mas ainda } \\
\text { apresentamos taxas elevadas de morte por } \\
\text { tais doenças. }\end{array}$ \\
\hline $\begin{array}{l}\text { Moreira et } \\
\text { al, } 2011\end{array}$ & $\begin{array}{l}\text { Estudo transversal com } \\
145 \text { professores }\end{array}$ & $\begin{array}{l}\text { Aferição da PAS de repouso, } \\
\text { medidas } \\
\text { mensuração antropométricas, } \\
\text { sanguíneos, dos parâmetros } \\
\text { componente cardiorrespiratório e } \\
\text { posterior elaboração de relatórios } \\
\text { individuais para cada avaliado, } \\
\text { utilização do teste ANOVA one } \\
\text { way e cálculo da razão de chances } \\
\text { de desenvolvimento de quadros de } \\
\text { PA elevada entre os indivíduos } \\
\text { que apresentavam níveis } \\
\text { indicativos de } \\
\text { cardiovascular. }\end{array}$ & $\begin{array}{l}\text { Os homens apresentaram valores significativamente maiores } \\
\text { para idade, peso, estatura, IMC, RCQ, CA, PAS e PAD. As } \\
\text { mulheres possuíam um percentual de gordura } \\
\text { significativamente elevado. Indivíduos com níveis } \\
\text { pressóricos elevados apresentaram valores maiores para } \\
\text { todas as variáveis, à exceção do VO2máx. Associação dos } \\
\text { fatores de risco e elevação da PA somente para as variáveis: } \\
\text { sexo, idade, IMC, CA, \%G e TG. }\end{array}$ & $\begin{array}{l}\text { O gênero, a idade, o IMC, a } \\
\text { circunferência abdominal, o percentual de } \\
\text { gordura corporal e os triacilgliceróis } \\
\text { foram considerados indicadores de risco } \\
\text { cardiovascular por apresentarem } \\
\text { associação com a hipertensão arterial na } \\
\text { amostra de professores avaliados. }\end{array}$ \\
\hline $\begin{array}{l}\text { Oliveira et } \\
\text { al, } 2009\end{array}$ & $\begin{array}{l}\text { Estudo transversal com } \\
180 \text { homens e } 120 \\
\text { mulheres. }\end{array}$ & $\begin{array}{l}\text { IMC, CC, percentual de gordura } \\
\text { corporal (\%G), relação cintura } \\
\text { quadril (RCQ), perfil lipídico, } \\
\text { glicemia e pressão arterial. }\end{array}$ & $\begin{array}{l}\text { IMC, CC e RCQ foram maiores nos homens e \%GC nas } \\
\text { mulheres. A proporção de casos alterados de RCQ e \%GC } \\
\text { em relação a LDL-c e CT foi maior no sexo masculino. } \\
\text { Correlação entre IMC e CC. Triglicerídeos (TG) teve } \\
\text { correlação com RCQ e com CC. }\end{array}$ & $\begin{array}{l}\text { O IMC e a RCQ foram os indicadores } \\
\text { antropométricos com maior correlação } \\
\text { com o perfil lipídico em ambos os sexos. } \\
\text { Hipótese de que o IMC e a RCQ podem } \\
\text { ser considerados como fatores de risco } \\
\text { para a doença cardiovascular. }\end{array}$ \\
\hline $\begin{array}{ll}\text { Pansani } & \text { et } \\
\text { al, } 2015 & \end{array}$ & $\begin{array}{l}\text { Foram divididos em } \\
\text { dois subgrupos } 91 \\
\text { indivíduos pela média } \\
\text { de índice de massa } \\
\text { corporal (IMC): Obesos } \\
\text { e Não Obesos }\end{array}$ & $\begin{array}{l}\text { Determinação da composição } \\
\text { corporal; Glicemia de jejum, } \\
\text { hemoglobina glicada e níveis } \\
\text { plasmáticos de aldosterona e } \\
\text { leptina. }\end{array}$ & $\begin{array}{l}\text { O subgrupo de obesos apresentou metabolismo da glicose } \\
\text { alterado pela glicemia de jejum e hemoglobina glicada. Os } \\
\text { níveis de aldosterona e leptina mais elevados em obesos. } \\
\text { Nível de glicose está independentemente associado à } \\
\text { obesidade em pacientes com hipertensão arterial resistente. }\end{array}$ & $\begin{array}{l}\text { O IMC pode ser determinante para } \\
\text { desregular o metabolismo da glicose, bem } \\
\text { como os níveis de aldosterona e leptina } \\
\text { em indivíduos hipertensos resistentes. }\end{array}$ \\
\hline
\end{tabular}




\begin{tabular}{|c|c|c|c|c|}
\hline $\begin{array}{l}\text { Rubio et al, } \\
2014\end{array}$ & $\begin{array}{l}\text { Análises de } 5.898 \\
\text { estudos } \\
\text { ecocardiográficos de } \\
\text { pacientes de idade entre } \\
18 \text { e } 92 \text { anos divididos } \\
\text { em } 6 \text { grupos segundo o } \\
\text { IMC e subdivisão de } \\
\text { idade e sexo, assim } \\
\text { como a prevalência da } \\
\text { comorbidade existente. }\end{array}$ & Ecocardiogramas e IMC. & $\begin{array}{l}\text { Na medida em que aumentou a massa corpórea aumentou } \\
\text { significativamente a prevalência de hipertensão arterial e } \\
\text { DM. }\end{array}$ & $\begin{array}{l}\text { Associação direta significativa entre o } \\
\text { aumento do IMC e o aumento da massa } \\
\text { miocárdica e uma associação inversa } \\
\text { entre o aumento da massa corpórea e a } \\
\text { relação E/A do enchimento mitral. } \\
\text { Aumento discreto, mas significativo, da } \\
\text { área e do volume indexado do átrio } \\
\text { esquerdo na medida em que aumenta a } \\
\text { massa corpórea. }\end{array}$ \\
\hline $\begin{array}{l}\text { Silva et al, } \\
2016\end{array}$ & 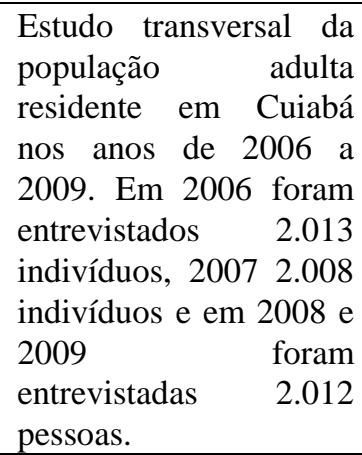 & $\begin{array}{l}\begin{array}{l}\text { Foram utilizadas informações } \\
\text { sobre características } \\
\text { sociodemográficas (sexo, idade e }\end{array} \\
\text { escolaridade) relacionadas ao } \\
\text { estilo de vida: tabagismo, uso de } \\
\text { bebida alcoólica, consumo } \\
\text { alimentar e atividade física. }\end{array}$ & $\begin{array}{l}\text { Aumento na proporção de homens que relataram nunca ter. } \\
\text { Redução do consumo regular de refrigerantes. Consumo de } \\
\text { frutas, legumes e verduras aumentou em } 19 \% \text {, mais evidente } \\
\text { em homens. Redução do relato de atividade física em } \\
\text { mulheres, porém, foi observado incremento na proporção de } \\
\text { mulheres e de indivíduos com mais de } 40 \text { anos de idade que } \\
\text { fazem deslocamento ativo diariamente. }\end{array}$ & $\begin{array}{l}\text { Os homens, os adultos jovens e os } \\
\text { indivíduos com nível educacional mais } \\
\text { elevado são os grupos que apresentam de } \\
\text { forma mais evidente variações favoráveis } \\
\text { relativas aos fatores de risco/proteção } \\
\text { para DCV. }\end{array}$ \\
\hline Soar, 2015 & $\begin{array}{lr}\text { Estudo transversal de } \\
955 \quad \text { idosos } \\
\text { frequentadores } & \text { do } \\
\text { Instituto Paulista de } \\
\text { Geriatria } & \text { e } \\
\text { Gerontologia em São } \\
\text { Paulo. }\end{array}$ & $\begin{array}{l}\text { Análise da prevalência de fatores } \\
\text { de risco cardiovasculares de } \\
\text { acordo com o sexo e grupo etário, } \\
\text { de Hipertensão Arterial, } \\
\text { Hipercolesterolemia, excesso de } \\
\text { peso e adiposidade abdominal. }\end{array}$ & $\begin{array}{l}\text { As prevalências de HAS, hipercolesterolemia, excesso de } \\
\text { peso e adiposidade abdominal foram, respectivamente, de } \\
82,30 \% \text { para idosas e } 76,81 \% \text { para idosos, } 43,64 \% \text { para } \\
\text { idosas e } 25,48 \% \text { para idosos, } 47,40 \% \text { para idosas e } 36,12 \% \\
\text { para idosos e de } 76,16 \% \text { para idosas e } 41,06 \% \text { para idosos. } \\
\text { Menor prevalência, somente para o excesso de peso, à } \\
\text { medida que a idade avança. }\end{array}$ & $\begin{array}{l}\text { As idosas são consideradas grupo mais } \\
\text { vulnerável para fatores de risco } \\
\text { cardiovascular, pois tiveram maior } \\
\text { número de medidas com valores acima do } \\
\text { recomendado, e prevalência de } \\
\text { hipertensão arterial, hipercolesterolemia, } \\
\text { excesso de peso e adiposidade. }\end{array}$ \\
\hline $\begin{array}{l}\text { Tacon et } \\
\text { al, } 2010\end{array}$ & $\begin{array}{lr}\text { Estudo } & \text { descritivo, } \\
\text { retrospectivo, } & \text { através } \\
\text { da análise } & \text { de } \\
\text { prontuários de pacientes } \\
\text { atendidos no período de } \\
\text { maio a julho de } & 2010 \text { no } \\
\text { Ambulatório r } & \text { de } \\
\text { Cardiologia no Hospital } \\
\text { de Urgências } \\
\text { Goiânia. de } \\
\text { analisados } & \text { Foram } \\
\text { prontuários. } & 103 \\
\end{array}$ & $\begin{array}{l}\text { O instrumento de pesquisa foi um } \\
\text { questionário estruturado com } \\
\text { dados sobre o perfil sócio- } \\
\text { demográfico, presença e } \\
\text { classificação da HAS, fatores de } \\
\text { risco associados, antecedentes } \\
\text { familiares e hábitos de vida desse } \\
\text { paciente. Foram considerados } \\
\text { hipertensos adultos acima de } 18 \\
\text { anos com diagnóstico de HAS em } \\
\text { tratamento. }\end{array}$ & $\begin{array}{l}\text { Os principais fatores de risco para DCV foram sedentarismo } \\
27 \% \text {, história de DCV na família } 20 \% \text {, idade acima de } 60 \\
\text { anos } 16 \% \text { e obesidade }(41,1 \%) \text {, índice de massa corpórea } \\
\text { de } 27-29 \text {. A maioria das pessoas em estágio } 3 \text { da HAS se } \\
\text { encontram em idade produtiva e não trabalha. }\end{array}$ & $\begin{array}{l}\text { A hipertensão arterial se manifesta } \\
\text { comumente na terceira idade, porém se } \\
\text { torna cada vez mais comum em jovens } \\
\text { portadores de doenças coronarianas por } \\
\text { se enquadrar em vários fatores de risco, } \\
\text { principalmente excesso de peso e } \\
\text { sedentarismo. Em relação à HAS, é, sem } \\
\text { dúvida, o principal fator de risco } \\
\text { modificável para DCV. }\end{array}$ \\
\hline
\end{tabular}




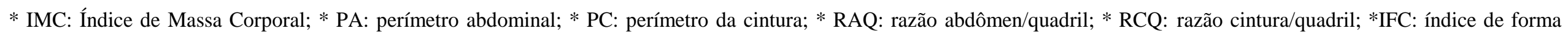

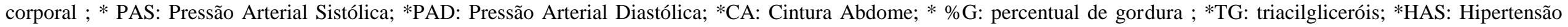

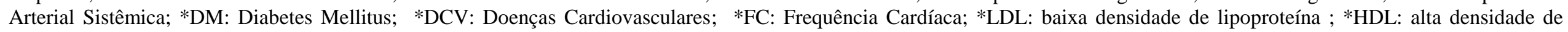

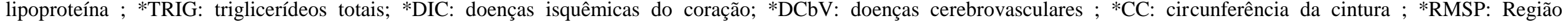
Metropolitana de São Paulo; *IBGE: Instituto Brasileiro de Geografia e Estatística 
A partir dos resultados obtidos percebese que o IMC elevado associado a outros fatores de risco para o sexo e a idade, revelam prejuízos na qualidade de vida dos indivíduos e aumentam a prevalência de doenças cardiovasculares. A idade mais avançada é fator preditor para hipertensão arterial, a qual é considerada o fator de risco com maior número de casos na população brasileira, assim como as baixas taxas de controle pressórico nos hipertensos (MANSUR et al, 2012).

Estudos epidemiológicos e de prevalência tem apresentado que não só houve prevalência da hipertensão arterial com a idade, também associada a fatores de risco, de estilo de vida e metabólicos, todos aumentando o risco para doenças cardiovasculares. Alterações glicídicas, lipídicas e pressóricas estão associados ao maior grau de obesidade (SOAR, 2015).

Segundo Mansur et al (2016), existe uma prevalência de doenças cardiovasculares nas regiões Norte, Centro-oeste, Sul, Sudeste e um aumento nos últimos anos na região Nordeste. A permanência da mortalidade nessas cinco regiões do Brasil levará em poucos anos a inversão do risco de morte entre elas, tornando a região Nordeste, e em menor grau as regiões Norte e Centro-Oeste, as regiões do Brasil com maiores índices de mortalidade por doenças cardiovasculares.

As principais limitações encontradas nos estudos e na literatura estão relacionadas à qualidade dos dados brasileiros de mortalidade como erros de diagnóstico e de atestado de óbitos, mortes relacionadas a causas desconhecidas e erros nas entradas de dados.
Além disso, estudos de validação para dados de taxa de mortalidade não são encontrados na grande maioria dos estados brasileiros, o que limita o estudo das populações.

\section{CONCLUSÃO}

Percebeu-se uma associação direta entre o IMC elevado como fator desencadeante de doenças cardiovasculares que, consequentemente, prejudicam a qualidade de vida. Esse estudo ratifica em grande medida o que previamente se tem descrito na literatura e aporta novo conhecimento útil para a compreensão do efeito do índice de massa corporal na relação com as doenças de origens cardiovasculares.

Percebe-se que os indicadores de risco cardiovascular foram o gênero, a idade, o IMC, a circunferência abdominal, o percentual de gordura corporal e os triacilgliceróis. Essa constatação denota a importância da inserção de políticas de promoção de saúde pautadas em ações especificas que atuem sobre os indicadores de risco cardiovascular e promovam melhor qualidade de vida.

\section{REFERÊNCIAS}

AUDI, C.A.F.; SANTIAGO, S.M.; ANDRADE, M.G.G.; FRANCISCO, P. M. S. B. Fatores de risco para doenças cardiovasculares em servidores de instituição prisional: estudo transversal. Epidemiologia e Serviços de Saúde, Brasília, 25(2):301-310, abr-jun 2016.

CARLETTI, L.; RODRIGUES, A.N.; PEREZ, A.J.; VASSALLO, D.V. Resposta da Pressão Arterial ao Esforço em Adolescentes: Influência do Sobrepeso e Obesidade. Revista Arquivos Brasileiros de Cardiologia 91(1):25-30, 2008. 
CHRISTOFARO, D.G.D.; ANDRADE, S.M.; FERNANDES, R.A.; OHARA, D.; DIAS, D.F.; JÚNIOR, I.F.F.; OLIVEIRA, D.R. Prevalência de fatores de risco para doenças cardiovasculares entre escolares em Londrina - PR: diferenças entre classes econômicas. Revista Brasileira de Epidemiologia, 14(1): 27-35, 2011.

COLTRO, R.S.; MIZUTANI, B.M.; MUTTI, A.; DÉLIA, M.P.B.; MARTINELLI, L.M.B.; COGNI, A.L.; MATSUBARA, B.B. Frequência de fatores de risco cardiovascular em voluntários participantes de evento de educação em saúde. Revista da Associação Médica Brasileira, 55(5): 606-10, 2009.

ESPINOSA, G.; PORTO, F.; GURGEL, J.L. Influência do Exercício Físico em Indicadores Antropométricos de Risco Cardiovascular em Idosas. Internacional Journal of Cardiovascular Sciences, 28(4):290-297, 2015.

FONSECA, H.A.R.; RECH, C.R.

Fatores de risco cardiovascular em mulheres da associação de obesos do município de Ponta Grossa-PR. Revista Arquivos Ciências da Saúde, 16(4):170-4 outdez, 2009.

LAVRADOR, M.S.F.; ABBES, P.T.; ESCRIVÃO, M.A.M.S.; TADDEI, J.A.A. Riscos Cardiovasculares em Adolescentes com Diferentes Graus De Obesidade. Revista Arquivos Brasileiros de Cardiologia.

96(3):205-211, 2011.

MANSUR, A.P.; FAVARATO, D.

Mortalidade por Doenças Cardiovasculares em Mulheres e Homens nas cinco Regiões do Brasil, 1980-2012. Arquivos Brasileiros de Cardiologia, [online].ahead print, PP.0-0, 2016.

MANSUR, A.P.; FAVARATO, D. Mortalidade por Doenças Cardiovasculares no Brasil e na Região Metropolitana de São Paulo: Atualização 2011. Revista Arquivos Brasileiros de Cardiologia 99(2): 755-761, 2012.

OLIVEIRA, M.A.M.; FAGUNDES, R.L.M.; MOREIRA, E.A.M.; TRINDADE, E.B.S.M.T; CARVALHO, TALES.

Relação de Indicadores Antropométricos com Fatores de Risco para Doença Cardiovascular.

Revista Arquivos Brasileiros de Cardiologia, [online]. ahead print, PP.0-0, 2010.

MOREIRA, O.C.; OLIVEIRA, R.A.R.; ANDRADE NETO, F.; AMORIM, W.; OLIVEIRA, C.E.P.; DOIMO, L.A.; AMORIM, P.R.S.; LATERZA, M.C.; MONTEIRO, W.D.; MARINS, J.C.B. Associação entre risco cardiovascular e hipertensão arterial em professores universitários. Revista brasileira de Educação Física e Esporte, São Paulo, v.25, n.3, p.397-406, jul./set. 2011.
PANSANI, I.F.; FARIA, A.P.C.; MODOLO, R.; MORENO, H. Body mass index as a determinant for metabolic-related changes in resistant hypertension. Revista Sociedade Brasileira de Clínica Médica, 13(1): 18-23, jan-mar, 2015.

RUBIO, L.D.C.S.; MOLINA, G.R.; ANAYA, M.L.B.; MARTÍNEZ, L.A.D.; TORRES, J.L.L.; PERILLA, K.E. Relação do Índice de Massa Corpórea com as Mudanças na Geometria e Função Cardíaca em 5.898 Pacientes Avaliados por Ecocardiografia Transtorácica. Revista Arquivos Brasileiros de Cardiologia. 28(1): 3-16, 2015.

SILVA, S.M.; LUIZ, R.R.; PEREIRA, R.A. Fatores de risco e proteção para doenças cardiovasculares em adultos de Cuiabá, Mato

Grosso, Brasil. Revista Brasileira de Epidemiologia, 18(2): 425-438, abr-jun, 2015.

SOAR, Claúdia. Prevalência de fatores de risco cardiovascular em idosos não institucionalizados. Revista Brasileira de Geriatria e Gerontologia, Rio de Janeiro, 18(2): 385-395 2015.

TACON, K.C.B.; SANTOS, H.C.O.; CASTRO, E.C. Perfil epidemiológico da hipertensão arterial sistêmica em pacientes atendidos em hospital público. Revista Brasileira de Clínica Médica, São Paulo, 8(6): 486-9, nov-dez, 2010.

\section{Letícia Carvalho Massaroli}

Discente do curso de Medicina do Centro Universitário das Faculdades Associadas de EnsinoUNIFAE.

\section{Letícia Cristina Santos}

Discente do curso de Medicina do Centro Universitário das Faculdades Associadas de EnsinoUNIFAE.

\section{Giovanna Gomes Carvalho}

Discente do curso de Medicina do Centro Universitário das Faculdades Associadas de EnsinoUNIFAE.

Stephanie Avesani João Figueiredo Carneiro
Discente do curso de Medicina do Centro
Universitário das Faculdades Associadas de Ensino-
UNIFAE

Laura Ferreira de Rezende

Mestre e Doutora pela Faculdade de Ciências Médicas da Universidade de Campinas e pós-doutora pela Universidade do Estado de São Paulo 